\title{
Platelet-rich Fibrin for Chronic Wounds Management
}

\section{Mena Zarif Helmy ${ }^{1}$, Mohammed Hussein Khalifa ${ }^{2}$, Asser AbdElhamed Goda ${ }^{2}$, Kamal A. A. M. Hasanein*1}

Departments of ${ }^{1}$ General Surgery and ${ }^{2}$ Vascular Surgery, Faculty of Medicine, Sohag University, Sohag, Egypt

*Corresponding author: Mohammed Hussein Khalifa, Mobile: (+20) 01019174312, Email: mohammedtammam28@ gmail.com

\begin{abstract}
Background: A chronic wound can be defined as any wound that fails to heal within a reasonable period. Platelet-rich fibrin (PRF) is a second-generation platelet concentrate. It is safe, simple, affordable and less expensive procedure in treatment of chronic wounds.
\end{abstract}

Objective: This study aimed to assess safety and effectiveness of platelet-rich fibrin for treatment of chronic wounds.

Patients and methods: This prospective study included 50 patients with chronic ulcer not responding to conventional therapy for at least 6 weeks, normal values blood tests especially platelet count, red blood cell count and serum albumin level. Debridement of the necrotic tissues was done using surgical and chemical debridement. The fibrin clot membrane was applied to the ulcer followed by paraffin gauze and secondary sterile dry dressing.

Results: Complete wound healing occurred in 18 of 50 patients before 8th week of therapy (36\%). A decrease in the mean area was observed from $7.22 \pm 5.80 \mathrm{~cm}^{2}$ before treatment to $0.98 \pm 1.52 \mathrm{~cm}^{2}$ after 8 weeks of therapy, which was highly significant.

Conclusions: The use of PRF is effective, simple, affordable and inexpensive method allowing acceleration of healing of chronic wounds.

Keywords: Platelet-rich Fibrin, Chronic Wounds, Diabetic foot ulcer, Critical limb ischemia.

\section{INTRODUCTION}

Chronic wounds exhibit such a disrupted repair process where a sustained anatomical and functional result is not reached within an appropriate length of time usually taken to be three months. They fail to progress through the orderly phases of healing where interruptions in the inflammatory phase during the normal wound healing process occurs ${ }^{(\mathbf{1})}$. Different factors can delay wound healing including chronic diseases, vascular insufficiency, diabetes, malnutrition, ageing and local factors such as pressure, infection, and edema ${ }^{(2)}$.

Chronic wounds have been associated with high costs and increased anxiety, both of which can negatively impact patients' quality of life ${ }^{(3)}$. The use of blood derivatives was often suggested in the last 50 years for the treatment of chronic skin wounds and the use of platelet-fibrin concentrates is one of approaches of regenerative medicine in modern therapy ${ }^{(4)}$. Fibrin matrix and platelet components (particularly growth factors) offer interesting healing properties as surgical adjuvants. Leukocyte- and platelet-rich fibrin (L-PRF) is an autologous blood-derived product, which can be obtained quickly and at low cost ${ }^{(4)}$.

The PRF, described by Choukroun ${ }^{(5)}$, is a new therapeutic approach using blood derivatives, with a simplified design and requiring minor artificial biochemical changes. This technique does not require anticoagulants, thrombin, or any other gelling agent, which renders it different from natural blood, centrifuged without additives ${ }^{(5)}$. PRF is useful in wound healing and skin regeneration as a primary and a supplemental technique owing to its fibrin matrix, cellular components, and prolonged release of growth factors is a feasible, safe, simple and inexpensive $\operatorname{method}^{\left({ }^{(6)} \text {. }\right.}$

This study aims to assess the safety and efficacy of platelet rich fibrin for treatment of chronic wounds.

\section{PATIENTS AND METHODS}

This was a prospective study that was conducted on patients with chronic wounds who were presented to our Outpatient Clinics of Vascular Surgery and General Surgery Departments, Faculty of Medicine, Sohag University.

\section{Inclusion criteria:}

Cases having an open wound for more than four weeks described as a chronic wound by a clinical professional, with no active bacterial, viral or fungal infection in the wound, wound area less than $50 \mathrm{~cm}^{2}$, not taking any anticoagulants or equivalent functional drugs for one month before enrolment and number of platelets in the range of 150,000 to $450,000 / \mathrm{UL}$ and coagulation index determined as normal [prothrombin time (PT value) ranging from 11 to 16 seconds]. Hemoglobin concentration $>9 \mathrm{mg} / \mathrm{dl}$, serum protein concentrations $>6 \mathrm{gram} / \mathrm{dl}$ and serum albumin $>3$ $\mathrm{gm} / \mathrm{dl}$.

\section{Exclusion criteria:}

Cases with wound area more than $50 \mathrm{~cm}^{2}$, ulcers with exposed bones, ligament or tendon, patients with peripheral arterial disease, characterized by ankle brachial index (ABI) less than 1, patients with systemic infection and/or clinical manifestations compatible with active infection of ulcer, irradiation, chemotherapy, malignant ulcers, terminal disease e.g. terminal stages of malignancy, uncontrolled systemic disease e.g. 
uncontrolled DM, current use of anticoagulants or immunosuppressant drugs and impaired coagulation profile, plasma protein level or HB concentration.

\section{All patients were subjected to the following:}

1. Full history taking from all patients including age and sex-history about the wound and its duration, history suggestive of peripheral ischemia, history of hypertension or hypotension, history of bleeding tendency or recurrent thrombosis and history of diabetes mellitus and its treatment. History of chronic disease, tumor or drug.

2. General examination: General condition, vital signs (pulse, blood pressure, temperature and respiration) and systemic examination.

3. Local examination: Complete examination of the four limbs including examination of the arterial pulsation, manifestation of arterial ischemia or venous congestion and examination of the wound site, size, infected or not infected, skin around the wound and sensation.

4. Routine investigation including $\mathrm{CBC}$, bleeding time and coagulation time, prothrombin time and concentration, serum proteins and albumin level, blood urea, serum creatinine and glycosylated $\mathrm{Hb}$ (Hb A1c).

\section{Platelet-rich fibrin (PRF) Technique:}

Before the application of PRF, all wounds were inspected carefully for any necrotic tissues that may cause poor healing. Debridement of the necrotic tissues was done using surgical and chemical debridement.

\section{Leucocyte-platelet rich fibrin (L-PRF) preparation:}

A blood sample was taken, with a volume depending on the wound area, without anticoagulant in 10 -ml tubes, immediately centrifuged at $3000 \mathrm{rpm}$ for $10 \mathrm{~min}$. Coagulation starts during the centrifugation, a fibrin clot was then obtained from the middle of the tube, and was transformed into a membrane circa $1 \mathrm{~mm}$ in thickness, by careful compression between two gauzes. The fibrin clot membrane was applied to the ulcer followed by paraffin gauze and secondary sterile dry dressing. Lastly, elastic bandage was applied. The frequency of change of dressing was once weekly. The dressing protocol was performed for up to 8 weeks or stopped whenever healing occurred.

\section{Follow up:}

We followed up the patients every week for at least 8 weeks or when healing was completed. Wound size (Length and width) was measured at initial visit (Initial size) and during each follow up visit weekly by measuring the ulcer's dimensions (Length and width) using sterile metalic ruler or metric tapes. At follow up visits: wound was examined for: wound size (length and width), presence of granulation tissue and presence of infection. Patients were examined for: presence of possible adverse effects as anemia, hypoalbuminemia. So, blood sample was taken from all patients at initial visit and every four weeks and was examined for complete blood count.

\section{Ethical approval:}

The study was approved by the Ethical Committee of Sohag Faculty of Medicine. Informed consents were obtained from all patients in this research. Every patient received an explanation for the purpose of the study. All given data were used for the current medical research only.

This work has been carried out in accordance with The Code of Ethics of the World Medical Association (Declaration of Helsinki) for studies involving humans.

\section{Statistical analysis}

Data were analyzed using STATA intercooled. Quantitative data was represented as mean $\pm \mathrm{SD}$, median and range. Paired values for planimetric data including wound area $\left(\mathrm{cm}^{2}\right)$ (length and width) were compared with paired samples among consecutive times of study period.

\section{RESULTS}

Table (1) showed baseline characteristics of the studied patients.

Regarding wound area $\left(\mathrm{cm}^{2}\right)$, that were performed between consecutive weeks, planimetric wound area showed significant reduction, also there were significant reductions among all consecutive measurements (Table 2).

Regarding reduction in mean wound area, in the $1^{\text {st }}$ week follow up there was significant reduction, also it was observed that reduction in mean wound area was highly significant (Table 3).

Hemoglobin and serum albumin were insignificantly different after the follow up compared to baseline. This means that there was no adverse effect for PRF as regards hemoglobin level and serum albumin (Table 4).

A male patient 36 years old, smoker, with posttraumatic ulcer 4 years duration, intact distal pulse showed an improvement and healing of ulcer (Figure 1). A second case; male patient 50 years with diabetic ulcer of 3 months duration, intact distal pulse observed an enhancement of healing ulcer after PRF sessions (Figure 1). 
Table (1): Baseline characteristics of the studied patients

\begin{tabular}{|l|c|c|}
\hline \multicolumn{1}{|c|}{ Variable } & $\begin{array}{c}\text { Number of } \\
\text { patients }\end{array}$ & Percentage of patients \\
\hline Age (years) & \multicolumn{2}{|c|}{$\begin{array}{c}44.18 \pm 11.80 \\
\text { (range 20 - 65) }\end{array}$} \\
\hline Gender: & 42 & $84 \%$ \\
- Males & 8 & $16 \%$ \\
- Females & 26 & $52 \%$ \\
\hline Diabetes & 10 & $20 \%$ \\
\hline Hypertension & 20 & $40 \%$ \\
\hline Smoking & 24 & $48 \%$ \\
\hline Diabetic foot ulcer & 22 & $44 \%$ \\
\hline Venous ulcer & 4 & $8 \%$ \\
\hline Traumatic ulcer & \multicolumn{2}{|c|}{4 (range 2-6) } \\
\hline Duration of chronic wound before PRF (months) & \multicolumn{2}{|c|}{$7.22 \pm 5.80 \pm 7414$} \\
\hline Mean platelet count & \multicolumn{2}{|c|}{} \\
\hline Mean wound area $\left(\mathrm{cm}^{2}\right)$ & \multicolumn{2}{|c|}{} \\
\hline
\end{tabular}

Table (2): wound area $\left(\mathrm{cm}^{2}\right)$ over study period in consecutive visits every week

\begin{tabular}{|l|c|c|}
\hline \multicolumn{1}{|c|}{ Study period } & $\begin{array}{c}\text { Wound area }(\mathbf{c m} \text { 2) } \\
\text { Mean } \pm \text { SD, Median (range) }\end{array}$ & $\begin{array}{c}\text { P compared to } \\
\text { previous study } \\
\text { period }\end{array}$ \\
\hline Before treatment & $7.22 \pm 5.80,6,(1-27.4)$ & $<0.0001^{*}$ \\
\hline $1^{\text {st }}$ week & $6.28 \pm 5.53,4.90,(0.5-27)$ & $<0.0001^{*}$ \\
\hline $2^{\text {nd }}$ week & $5.38 \pm 5.05,4.10,(0.39-25.6$ & $<0.0001^{*}$ \\
\hline $3^{\text {rd }}$ week & $4.49 \pm 4.53,3.15,(0.18-23)$ & $<0.0001^{*}$ \\
\hline $4^{\text {th }}$ week & $3.64 \pm 3.91,2.45,(0.1-19.3)$ & $<0.0001^{*}$ \\
\hline $5^{\text {th }}$ week & $2.79 \pm 3.09,1.85,(0.1-13.7)$ & $<0.0001^{*}$ \\
\hline $6^{\text {th }}$ week & $2.17 \pm 2.59,1.45,(0-10.2)$ & $<0.0001^{*}$ \\
\hline $7^{\text {th }}$ week & $1.59 \pm 2.09,1,(0-8.7)$ & $<0.0001^{*}$ \\
\hline $8^{\text {th }}$ week & $0.98 \pm 1.52,0.40,(0-6.3)$ & \\
\hline
\end{tabular}

Table (3): Reduction in mean wound area and its percent in consecutive visits

\begin{tabular}{|l|c|c|}
\hline \multicolumn{1}{|c|}{ Study period } & $\begin{array}{c}\text { Reduction in mean wound area } \\
\text { cm2 }\end{array}$ & $\begin{array}{c}\text { Percent of reduction in mean } \\
\text { wound area }\end{array}$ \\
\hline 1st week & 0.94 & $13 \%$ \\
\hline 2nd week & 1.84 & $25 \%$ \\
\hline 3rd week & 2.73 & $37.8 \%$ \\
\hline 4th week & 3.58 & $49.58 \%$ \\
\hline 5th week & 4.25 & $58.86 \%$ \\
\hline 6th week & 5.05 & $69.94 \%$ \\
\hline 7th week & 5.63 & $77.97 \%$ \\
\hline 8th week & 6.24 & $86.42 \%$ \\
\hline
\end{tabular}

Table (4): Comparison between baseline and follow up hemoglobin and serum albumin were as following

\begin{tabular}{|l|l|c|c|c|}
\hline \multicolumn{2}{|l|}{ Variable } & Baseline & After follow up & P value \\
\hline Hemoglobin $(\mathrm{g} / \mathrm{dL})$ & Mean \pm SD & $11.85 \pm 0.97$ & $11.50 \pm 0.99$ & 0.174 \\
\hline Serum albumin (g/L) & Mean \pm SD & $3.75 \pm 0.45$ & $3.68 \pm 0.39$ & 0.405 \\
\hline
\end{tabular}




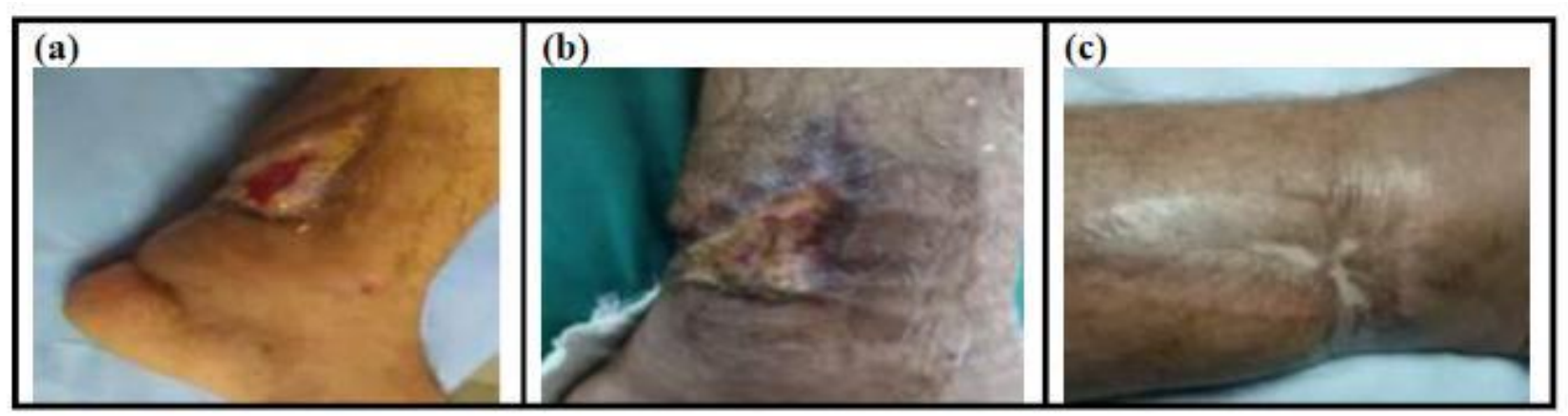

Figure (1): Male patient, 36 years old with post-traumatic ulcer showed (a) initial wound size, (b) after 3 sessions PRF and (c) After $6^{\text {th }}$ session PRF

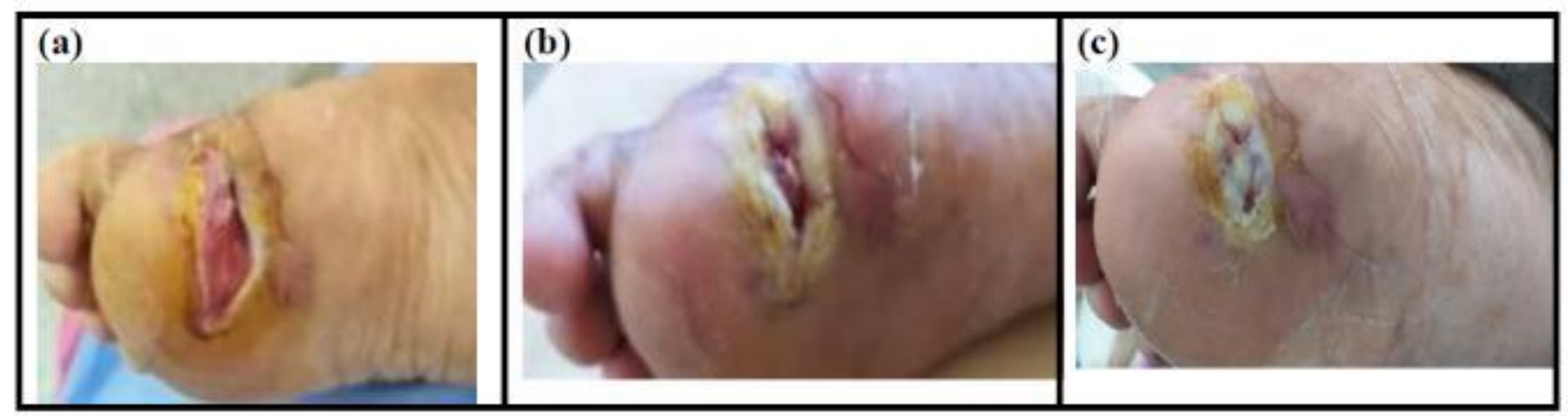

Figure (2): Male patient, 50 years with diabetic ulcer of 3 months duration showed (a) Initial wound size, (b) after 4 sessions PRF and (c) after 6th weeks of PRF treatment.

\section{DISCUSSION}

Chronic wounds are a fairly frequent complication. It has been linked to higher expenses and anxiety. Both of which may have a detrimental effect on patients' quality of life ${ }^{(7)}$.

The premise for this research stems from the growing interest in the use of regenerative medicine in contemporary medicine for the treatment of persistent wounds when traditional therapy fails to produce acceptable healing outcomes. PRF includes a high concentration of PDGF, VEGF, and transforming growth factor (TGF-). All of which promote cell proliferation and differentiation, culminating in tissue development ${ }^{(5)}$.

In agreement with Goda ${ }^{(8)}$ and Pinto et al. ${ }^{(4)}$, a standardized PRF protocol for the treatment of chronic wounds comprised of blood samples collected without anticoagulant in $10-\mathrm{ml}$ vials and promptly centrifuged at $3000 \mathrm{rpm}$ for 10 minutes. During centrifugation, coagulation started, and three different components appeared in the tube: a red blood cell base at the bottom, cellular plasma in the supernatant (platelet-depleted plasma), and the L-PRF clot in the center. The latter is tightly packed with fibrin, platelets (which account for about $95 \%$ of the original blood), and leukocytes (50 percent of initial blood). After extracting a fibrin clot from the tube's center, the clot was gently squeezed between two gauzes to form a membrane approximately $1 \mathrm{~mm}$ thick. Once a week, dressings were changed.

The findings of this research are similar to those of Somani and Rai ${ }^{(9)}$ who included 15 people suffering with severe leg ulcers who had not responded to existing treatment modalities for more than six months. The ulcer was at least $1 \mathrm{~cm}^{2}$ in size and less than or equal to $5 \mathrm{~cm}^{2}$ in size. Patients were randomly assigned to one of two dressing groups: PRF dressing or saline dressing. According to that research, the mean decrease in the area of the ulcer size was 85.51 percent in the PRF group, which was statistically significant at $\mathrm{P} 0.001^{(9)}$. In contrast to a research conducted by Frykberg et al. ${ }^{(10)}$, which revealed a substantial decrease in the area of 63 of 65 ulcers of various etiologies after PRF therapy. This study demonstrated a significant reduction in the area of the ulcers following PRF treatment. Eleven out of 65 ulcers were venous ulcers that responded to therapy with a substantial decrease in the mean percentage of area (43.1\%) in 2.3 weeks, while in this research, the mean wound area was reduced by $25 \%$ in the second week and $37.8 \%$ in the third week. The findings of this research are less conclusive than those of Goda's (8) randomized-controlled trial, which included 36 patients with VUs. In this study, eligible patients were randomly assigned to one of two groups (PRF or control) based on the randomization schedule. Each group consisted of eighteen patients. After 1, 2, 3, $4,5,6$, and 7 weeks, the mean ulcer area decreased in the PRF group with an initial size more than $10 \mathrm{~cm}$ was $16.3,31.1,49.4,64.3,79.5$, and $96.3 \%$ respectively. This may be because $48 \%$ of cases in our research were diabetic foot ulcers, and some patients had uncontrolled diabetes that takes years to cure. However, in the Goda research ${ }^{(8)}$, all instances were venous ulcers. 
The results of this research are less than those of Pravin et al. (11), who conducted a randomised-openlabel comparative trial in which they included 30 patients with non-healing ulcers of different etiologies. Fifteen patients received L-PRF at weekly intervals for a maximum of six treatments. It demonstrated a greater than $90 \%$ reduction in ulcer area in 13 cases (86.6 percent of cases) and a 71-80\% reduction in wound area in the remaining two cases from the 15 cases treated with PRF at the end of the sixth week. Whereas, our study demonstrated a $69.94 \%$ reduction in wound area in all 50 cases at the end of the sixth week. This may be explained by the fact that just 2 of 15 patients in this research were diabetic, compared to 26 of 50 patients in our study. This may also be explained by the fact that the wound size in this research was not recorded, while in our investigation, some patients had wounds up to 25 $\mathrm{cm}^{2}$.

Our research had several limitations, such as a limited sample size and patient selection, which restricts the generalizability of our findings. Additionally, the single institution setting and the absence of a control group were significant limitations.

\section{CONCLUSION}

The use of PRF is effective, simple, affordable and inexpensive method allowing acceleration of healing of chronic wounds.

\section{Financial support and sponsorship: Nil. \\ Conflict of interest: Nil.}

\section{REFERENCES}

1. Atkin L (2019): Chronic wounds: the challenges of appropriate management. Br J Community Nurs., 24: 2632.
2. Bjarnsholt T, Kirketerp-Møller K, Jensen $P$ et al. (2008): Why chronic wounds will not heal: a novel hypothesis. Wound Repair and Regeneration, 16: 2-10.

3. Bryant R, Nix D (2015): Acute and chronic wounds-Ebook: Elsevier Health Sciences. https://www.goodreads.com/book/show/26514408acute-chronic-wounds

4. Pinto N, Ubilla M, Zamora Y et al. (2018): Leucocyteand platelet-rich fibrin (L-PRF) as a regenerative medicine strategy for the treatment of refractory leg ulcers: a prospective cohort study. Platelets, 29: 468-75.

5. Crisci A, Marotta G, Licito A et al. (2018): Use of Leukocyte Platelet (L-PRF) Rich Fibrin in Diabetic Foot Ulcer with Osteomyelitis (Three Clinical Cases Report). Diseases, 6 (2): 30-35.

6. Bakker K, Apelqvist J, Schaper $\mathrm{N}$ et al. (2012): Practical guidelines on the management and prevention of the diabetic foot 2011. Diabetes/Metabolism Research and Reviews, 28: 225-31.

7. Nunan R, Harding K, Martin P (2014): Clinical challenges of chronic wounds: searching for an optimal animal model to recapitulate their complexity. Dis Model Mech., 7: 1205-1213.

8. Goda A (2018): Autogenous leucocyte-rich and plateletrich fibrin for the treatment of leg venous ulcer: A randomized control study. Egypt J Surg., 37: 316-9.

9. Somani A, Rai R (2017): Comparison of efficacy of autologous platelet-rich fibrin versus saline dressing in chronic venous leg ulcers: A randomised controlled trial. Journal of Cutaneous and Aesthetic Surgery, 10: 8-12.

10. Frykberg R, Banks J (2015): Challenges in the treatment of chronic wounds. Advances in Wound Care, 4: 560-82.

11. Pravin A, Sridhar V, Srinivasan B (2016): Autologous platelet rich plasma (PRP) versus leucocyte-platelet rich fibrin (L-PRF) in chronic non-healing leg ulcers- a randomised, open labelled, comparative study. J Evol Med Dent Sci., 5: 7460-3. 